\title{
GAMOW VECTORS IN THE BAKAMJIAN-THOMAS CONSTRUCTION
}

\author{
SUJEEV WICKRAMASEKARA
}

Communicated by Arno Bohm

\begin{abstract}
We show that the Bakamjian-Thomas construction for relativistic direct interactions can be extended to describe resonances by irreducible representations of the causal Poincaré semigroup. These representations are generated by a Poincaré algebra that incorporates interactions and are characterized by the complex pole position and spin of the resonance.
\end{abstract}

\section{Inroduction}

Unitary representations of the Poincaré group $\mathcal{P}$ underlie all physical theories that obey the principles of special relativity and quantum mechanics. The Poincaré group is also fundamental to classical relativistic physics. In this context, Dirac considered the problem of describing interactions between classical relativistic particles [4]. He worked within the framework of a Poincaré algebra realized by Poisson brackets of dynamical variables and did not make ancillary assumptions such as the existence of fields that mediate the interactions. In classical relativistic mechanics, Dirac's problem does not have a non-trivial solution if the particles are subjected to the (unphysical) constraint of having covariant world-lines. Worldline constraints do not certainly hold in the quantum setting, and Bakamjian and Thomas (BT) gave the first explicit construction of a class of relativistic quantum direct interaction theories for two particles [2]. The key theoretical idea of this work, which we will refer to as the BT construction, is that interactions can be introduced as a perturbation to the invariant mass operator $M=M_{0}+\Delta M$, much like in the non-relativistic case where the Hamiltonian absorbs the interactions, $H=H_{0}+V$. Interaction incorporating self-adjoint operators that furnish a realization of the Poincaré algebra can be induced from this interacting mass operator $M$ and the interaction-free two particle generators of the Poincare transformations. Here, it is possible to choose a kinematic subalgebra that remains unaffected by the interactions, leading to different forms of dynamics [4]. Sokolov 
extended the BT construction to more than two particles, the number of which is conserved or not, in a manner that satisfies the principle of cluster decomposition [13]. See [6] for the development of relevant ideas and [8] for an excellent comprehensive review of the subject and some interesting original results.

The purpose of this paper is to examine how the BT construction may be extended for the description of quasistable states. To illustrate the basic ideas, we consider the scattering of two stable particles leading to the formation of a resonance. The main technical result we report is the existence of an irreducible representation of the causal Poincaré semigroup, the semidirect product of the Lorentz group and the semigroup of spacetime translations into the forward lightcone, that describes the resonance. This representation is characterized by the position of the $S$-matrix resonance pole and the spin-value of the partial wave in which the pole appears. Thus, a state vector description with well-defined transformation properties under symmetry operations appears possible for a resonance, and the spacetime translations of these state vectors lead to a fundamental criterion by which unique mass and width values of the resonance can be extracted from the pole position.

\section{Bakamjian-Thomas Construction}

The Hilbert space $\mathcal{H}^{n}(m, s)$ of a stable elementary quantum system of mass $m$, spin $s$ and species labels $n$ furnishes a unitary, irreducible representation (UIR) of $\mathcal{P}$ [16]. Under the operators $U(\Lambda, a),(\Lambda, a) \in \mathcal{P}$, of this UIR, the generalized eigenvectors $\left|\boldsymbol{p}, s_{3}[m, s] n\right\rangle$ transform as

$$
U(\Lambda, a)\left|\boldsymbol{p}, s_{3}[m, s] n\right\rangle=\mathrm{e}^{-\mathrm{i} a \cdot p} \sum_{s_{3}^{\prime}} D_{s_{3}^{\prime} s_{3}}^{s}\left(W\left(\Lambda^{-1}, p\right)\right)\left|\boldsymbol{\Lambda} \boldsymbol{p}, s_{3}^{\prime}[m, s] n\right\rangle
$$

where $\boldsymbol{\Lambda} \boldsymbol{p}$ is the spatial part of the four vector $\Lambda p$ and $W(\Lambda, p)=L^{-1}(\Lambda p) \Lambda L(p)$ is a Wigner rotation. The vectors $\left|\boldsymbol{p}, s_{3}[m, s] n\right\rangle$ are not elements of the Hilbert space $\mathcal{H}^{n}(m, s)$ and they should be defined as continuous functionals on a proper subspace (test functions) of $\mathcal{H}(m, s)$. Below, we will consider test function spaces for two particle scattering.

The Hilbert space of two particles is the direct product

$$
\mathcal{H}=\mathcal{H}^{n_{1}}\left(m_{1}, s_{1}\right) \otimes \mathcal{H}^{n_{2}}\left(m_{2}, s_{2}\right) .
$$

The direct product operators $U_{0}(\Lambda, a)=U_{1}(\Lambda, a) \otimes U_{2}(\Lambda, a)$, where $U_{i}(\Lambda, a)$ are defined by (1) for the particle of mass $m_{i}$ and spin $s_{i}, i=1,2$, furnish a unitary 
representation of $\mathcal{P}$ in $\mathcal{H}(2)$. The Poincaré algebra that integrates to this direct product representation is spanned by the sum of one particle operators

$$
P_{0 \mu}:=P_{\mu}^{(1)}+P_{\mu}^{(2)}, \quad J_{0 \mu \nu}:=J_{\mu \nu}^{(1)}+J_{\mu \nu}^{(2)} .
$$

The subscript 0 in (3) and in the representation $U_{0}$ indicates that the two particles are free. Operators (3) fulfill the commutation relations of the Poincaré algebra

$$
\begin{aligned}
{\left[J_{0 \mu \nu}, J_{0 \rho \sigma}\right] } & =\mathrm{i}\left(g_{\nu \rho} J_{0 \mu \sigma}-g_{\mu \rho} J_{0 \nu \sigma}+g_{\mu \sigma} J_{0 \nu \rho}-g_{\nu \sigma} J_{0 \mu \rho}\right) \\
{\left[P_{0 \mu}, P_{0 \nu}\right] } & =0, \quad\left[P_{0 \mu}, J_{0 \rho \sigma}\right]=\mathrm{i}\left(g_{\mu \rho} P_{0 \sigma}-g_{\mu \sigma} P_{0 \rho}\right)
\end{aligned}
$$

The central elements are the mass and spin operators,

$$
M_{0}^{2}=P_{0 \mu} P_{0}^{\mu}, \quad W_{0}=\frac{1}{M_{0}^{2}} \omega_{0 \mu} \omega_{0}^{\mu}
$$

where $\omega_{0 \mu}=\frac{1}{2} \epsilon_{\mu \nu \rho \sigma} P_{0}^{\nu} J_{0}^{\rho \sigma}$. Unlike the generators (3), $M_{0}$ and $W_{0}$ are not the sums of the one particle operators.

The representation $U_{0}=U_{1} \otimes U_{2}$ in (2) is not irreducible, but it can be reduced to a direct sum of UIRs [10]

$$
\mathcal{H}=\mathcal{H}^{n_{1}}\left(m_{1}, s_{1}\right) \otimes \mathcal{H}^{n_{2}}\left(m_{2}, s_{2}\right)=\int_{\mathrm{s}_{0}}^{\infty} \mathrm{ds} \sum_{j \eta} \mathcal{H}^{\eta}(\mathrm{s}, j)
$$

where the integration is over the spectrum of $M_{0}^{2}, \mathrm{~s}_{0} \equiv\left(m_{1}+m_{2}\right)^{2} \leq \mathrm{s}<\infty$, the total angular momentum $j=0,1,2, \cdots$ if $\left|s_{1}-s_{2}\right|=$ integer, and $j=$ $1 / 2,3 / 2, \cdots$ if $\left|s_{1}-s_{2}\right|=$ half-odd-integer where $\eta$ represents the degeneracy labels that include orbital angular momentum $l$, total spin $s$ and particle species indices $n$. Each Hilbert subspace $\mathcal{H}^{\eta}(\mathrm{s}, j)$ of (6) furnishes a UIR of $\mathcal{P}$ corresponding to mass $\sqrt{\mathrm{s}}$ and $\operatorname{spin} j$. The generalized eigenvectors for this UIR $\left|\boldsymbol{p}, j_{3}[\mathrm{~s}, j] \eta\right\rangle$, which can be determined from the direct product basis vectors by means of the Clebsch-Gordan coefficients, transform irreducibly under $\mathcal{P}$.

Let us turn to the problem of introducing interactions into the above two particle system along the lines of BT construction. To that end, consider a perturbation of the mass operator (5) of the form

$$
M:=M_{0}+\Delta M
$$

The central idea is to construct a set of ten operators $P_{\mu}$ and $J_{\mu \nu}$ such that the commutation relations (4) are fulfilled and the defining relation $M=P_{\mu} P^{\mu}$ holds 
for the $M$ defined by (7). Here we choose the point form dynamics [4]. In terms of generators, this means that only the momenta are affected by the interactions. Thus, we define the interacting operators $P_{\mu}$ and $J_{\mu \nu}$ by

$$
P_{\mu}=M \frac{P_{0} \mu}{M_{0}}=P_{0 \mu}+\Delta M \frac{P_{0 \mu}}{M_{0}}, \quad J_{\mu \nu}=J_{0 \mu \nu} .
$$

To ensure relativistic invariance of the interacting system, it is necessary that operators (8) fulfill the commutation relations (4). The first equality of (4) is automatic since $J_{\mu \nu}=J_{0 \mu \nu}$ while the last two require $\left[\frac{P_{0 \mu}}{M_{0}}, \Delta M\right]=0$ and $\left[J_{0 \mu \nu}, \Delta M\right]=0$. This means that the interaction term $\Delta M$ must be a function of only the internal variables, such as the magnitude of the relative momentum $k$ and the degeneracy labels $\eta$ (including $l$ and $s$ ). It follows from (8) that the spin operator $W=\frac{1}{M^{2}} \omega_{\mu} \omega^{\mu}$, where $\omega_{\mu}=\frac{1}{2} \epsilon_{\mu \nu \rho \sigma} P^{\nu} J^{\rho \sigma}$, is identical to the free operator $W_{0}$ of (5). From these considerations we see that the BT construction provides a way of inducing perturbations into the 10-parameter group $\mathcal{P}$ from the well established theory of perturbations for the one dimensional Lie group $\mathbb{R}$.

Under the usual integrability conditions for operator Lie algebras [11], the interacting Lie algebra (8) integrates to a unitary representation of $\mathcal{P}$ in (2). We denote this unitary representation by $U$, as opposed to $U_{0}$, the one generated by the free operators (3).

\section{Resonance Scattering and Decay}

In order to describe the interacting two body system, in particular scattering, we must solve the eigenvalue problem for a complete system of commuting observables (CSCO) in the enveloping algebra generated by the operators (8). In anticipation of scattering, we will assume that the interaction $\Delta M$ is such that the spectrum of the full mass operator $M$ is absolutely continuous and coincides with that of the free mass operator $M_{0}$. We will further assume that asymptotic completeness holds and Møller operators exist, $\Omega_{ \pm}=\lim _{\tau \rightarrow \mp \infty} \mathrm{e}^{\mathrm{i} M \tau} \mathrm{e}^{-\mathrm{i} M_{0} \tau}$. Operators $\Omega_{ \pm}$map the Hilbert space (2) unitarily onto itself such that, for $\varphi \in \mathcal{H}, \Omega_{+} \varphi:=$ $\phi^{+}$are the scattering in-vectors and $\Omega_{-} \varphi:=\psi^{-}$are the out-vectors. They also map the generalized eigenvectors $\left|\boldsymbol{p}, j_{3}[\mathrm{~s}, j] \eta\right\rangle$ into interacting in- and out- generalized eigenvectors: $\left|\boldsymbol{p} j_{3}[s, j] \eta^{ \pm}\right\rangle=\Omega_{ \pm}\left|\boldsymbol{p}, j_{3}[\mathrm{~s}, j] \eta\right\rangle$. Like $\left|\boldsymbol{p}, j_{3}[\mathrm{~s}, j] \eta\right\rangle$, the vectors $\left|\boldsymbol{p}, j_{3}[\mathbf{s}, j] \eta^{ \pm}\right\rangle$must be properly defined as continuous antilinear functionals on a suitable (test functions) subspaces of the Hilbert space (2). We will shortly see that the analyticity properties of the $S$-matrix provide the clues as to how the relevant subspaces of $\mathcal{H}$ are to be constructed. When so defined as functionals, the 
vectors $\left|\boldsymbol{p}, j_{3}[\mathbf{s}, j] \eta^{+}\right\rangle$and $\left|\boldsymbol{p}, j_{3}[\mathbf{s}, j] \eta^{-}\right\rangle$furnish a basis for the in- vectors $\phi^{+}$and out- vectors $\psi^{-}$, respectively. Therewith, the $S$-matrix element has the expansion

$$
\left\langle\psi^{-} \mid \phi^{+}\right\rangle=\sum_{j j_{3} \eta_{\mathbf{s}_{0}}} \int_{\mathrm{d}}^{\infty} \mathrm{d} \mathbf{s} \frac{\mathrm{d} \boldsymbol{p}}{2 p^{0}}\left\langle\psi^{-} \mid \boldsymbol{p}, j_{3}[\mathbf{s}, j] \eta^{-}\right\rangle S_{j}(\mathbf{s})\left\langle\boldsymbol{p}, j_{3}[\mathbf{s}, j] \eta^{+} \mid \phi^{+}\right\rangle
$$

where the reduced $S$-matrix $S_{j}(\mathrm{~s})$ is defined by

$$
\left\langle\boldsymbol{p}^{\prime}, j_{3}^{\prime}\left[\mathbf{s}^{\prime}, j^{\prime}\right] \eta^{\prime-} \mid \boldsymbol{p}, j_{3}[\mathbf{s}, j] \eta^{+}\right\rangle=\delta\left(\boldsymbol{p}^{\prime}-\boldsymbol{p}\right) \delta\left(\mathrm{s}^{\prime}-\mathrm{s}\right) \delta_{j^{\prime} j} \delta_{j_{3}^{\prime} j_{3}} \delta_{\eta^{\prime} \eta} S_{j}(\mathrm{~s}) .
$$

If a resonance forms, then the analytic function $S_{j=j_{R}}(\mathrm{~s})$, where $j_{R}$ is the spin of the resonance, has a pair of simple poles at $\mathrm{s}=\mathrm{s}_{R}$ and its complex conjugate $\mathrm{s}=\mathrm{s}_{R}^{*}$ in the second Riemann sheet [5]. Let us take $\operatorname{Im}\left(\mathrm{s}_{R}\right)<0$. For the sake of simplicity, let us also take $s_{1}=s_{2}=0$ and the spin of the resonance $j_{R}=0$. Therewith, we consider only the $j=0$ term in (9).

In order to bring forth the contribution of the resonance, we must consider the extension of the integral over $s$ in (9) into one defined over a contour that encircles the pole position $\mathrm{s}=\mathrm{s}_{R}$ on the lower half plane. This in turn requires that the integrand of (9) have an analytic extension in s. Since $S_{0}(\mathrm{~s})$ is already an analytic function, we only need to demand that the wave functions $\left\langle\psi^{-} \mid \boldsymbol{p}[\mathrm{s}, 0]^{-}\right\rangle$ and $\left\langle\boldsymbol{p}[\mathrm{s}, 0]^{+} \mid \phi^{+}\right\rangle$have analytic extensions into the lower half complex s-plane. This means that we must introduce boundary conditions into the wave functions in addition to the usual square integrability.

Since $p_{\mu} p^{\mu}=\mathrm{s}$, complex extensions of wavefunctions in s necessarily leads to complex extensions in $\boldsymbol{p}$. However, complications of having to deal with functions of multiple complex variables can be avoided if we label the generalized eigenvectors by the spatial part of the four velocity $q=\frac{p}{\sqrt{s}}$, instead of the momenta $\boldsymbol{p}$. This choice introduces no loss of generality since it amounts to choosing the set $\left\{\frac{P_{\mu}}{M}, S_{3}, M, W\right\}$, rather than $\left\{P_{\mu}, S_{3}, M, W\right\}$, as a CSCO. For the CSCO $\left\{\frac{P_{\mu}}{M}, S_{3}, M, W\right\}$ and $j=0$, the $S$-matrix has the expansion

$$
\left\langle\psi^{-} \mid \phi^{+}\right\rangle=\int_{\mathbf{s}_{0}}^{\infty} \mathrm{ds} \int \frac{\mathrm{d} \boldsymbol{q}}{2 q^{0}}\left\langle\psi^{-} \mid \boldsymbol{q}[\mathbf{s}, 0]^{-}\right\rangle S_{0}(\mathrm{~s})\left\langle\boldsymbol{q}[\mathbf{s}, 0]^{+} \mid \phi^{+}\right\rangle .
$$

The velocity eigenvectors have the normalization $\left\langle\boldsymbol{q}^{\prime}, j_{3}^{\prime}\left[\mathbf{s}^{\prime}, j^{\prime}\right] \eta^{\prime \pm} \mid \boldsymbol{q}, j_{3}[\mathbf{s}, j] \eta^{ \pm}\right\rangle=$ $2 q^{0} \delta\left(\mathbf{s}^{\prime}-\mathbf{s}\right) \delta\left(\boldsymbol{q}^{\prime}-\boldsymbol{q}\right) \delta_{j_{3}^{\prime} j_{3}} \delta_{j^{\prime} j} \delta_{\eta^{\prime} \eta}$, so that $\left\langle\boldsymbol{q}^{\prime}, j_{3}^{\prime}\left[\mathbf{s}^{\prime}, j^{\prime}\right] \eta^{\prime-} \mid \boldsymbol{q}, j_{3}[\mathbf{s}, j] \eta^{+}\right\rangle=2 q^{0} \delta\left(\mathbf{s}^{\prime}-\right.$ s) $\delta\left(\boldsymbol{q}^{\prime}-\boldsymbol{q}\right) \delta_{j_{3}^{\prime} j_{3}} \delta_{j^{\prime} j} \delta_{\eta^{\prime} \eta} S_{j}(\mathbf{s})$. The velocity wave functions $\left\langle\psi^{-} \mid \boldsymbol{q}, j_{3}[\mathbf{s}, j]^{-}\right\rangle$and $\left\langle\boldsymbol{q}, j_{3}[\mathbf{s}, j]^{+} \mid \phi^{+}\right\rangle$have the advantage that they can be analytically extended in the 
square mass $s$ while keeping the velocity variables real. This leads complex momenta of the form $\sqrt{s} q_{\mu}$ where $q_{\mu}$ are real.

Recall that the Hilbert space for the representation $U$ can be always realized as $L^{2}$-functions defined on the Cartesian product of the spectra of a CSCO. For the $\operatorname{CSCO}\left\{\frac{P_{\mu}}{M}, S_{3}, M, W\right\}$ and $j=0$, the $L^{2}$-realization of $\mathcal{H}_{j=0}=\int_{\mathrm{s}_{0}} \mathrm{ds} \mathcal{H}(\mathrm{s}, 0)$ is $L^{2}\left(\mathbb{R}_{\mathrm{s}_{0}}, \mathbb{R}^{3}\right)$, where $\mathbb{R}_{\mathrm{s}_{0}}$ is the spectrum of $M^{2}$. Out of these $L^{2}$-functions, we must choose for $\left\langle\psi^{-} \mid \boldsymbol{q}[\mathrm{s}, 0]^{-}\right\rangle$and $\left\langle\boldsymbol{q}[\mathrm{s}, 0]^{+} \mid \phi^{+}\right\rangle$those which admit analytic extensions into the lower half complex plane. Further, for the contour deformation of the integral (10) to be defined, these analytic extensions must decrease in the modulus sufficiently fast for $|\mathbf{s}| \rightarrow \infty$. These requirements can be fulfilled if we choose $\left\langle\psi^{-} \mid \boldsymbol{q}[\mathrm{s}, 0]^{-}\right\rangle$and $\left\langle\boldsymbol{q}[\mathrm{s}, 0]^{+} \mid \phi^{+}\right\rangle$to be of Hardy class [9] from below in the square mass variable s. Specifically, let

$$
\begin{aligned}
& \left\{\left\langle\boldsymbol{q}[\mathrm{s}, 0]^{-} \mid \psi^{-}\right\rangle\right\}=\left.\mathcal{M} \cap \mathcal{H}_{+}^{2}\right|_{\mathbb{R}_{\mathrm{s}_{0}}} \otimes \mathcal{S}\left(\mathbb{R}^{3}\right) \\
& \left\{\left\langle\boldsymbol{q}[\mathrm{s}, 0]^{+} \mid \phi^{+}\right\rangle\right\}=\left.\mathcal{M} \cap \mathcal{H}_{-}^{2}\right|_{\mathbb{R}_{\mathrm{s}_{0}}} \otimes \mathcal{S}\left(\mathbb{R}^{3}\right) .
\end{aligned}
$$

Here, $\mathcal{S}\left(\mathbb{R}^{3}\right)$ is the Schwartz space over $\mathbb{R}^{3}, \mathcal{H}_{ \pm}^{2}$ are Hardy class functions on $\mathbb{C}^{ \pm}$, and $\mathcal{M}$ is the subspace of Schwartz functions which, along with all of their derivatives, vanish at the origin. The symbol $\left.\right|_{\mathbb{R}_{s_{0}}}$ indicates the restrictions of the functions in $\mathcal{M} \cap \mathcal{H}_{ \pm}^{2}$, the support of which is the whole real line, to the spectrum of $M^{2}$. Since $\left\langle\boldsymbol{q}[\mathrm{s}, 0]^{-} \mid \psi^{-}\right\rangle \in \mathcal{H}_{+}^{2}$ implies $\overline{\left\langle\boldsymbol{q}[\mathrm{s}, 0]^{-} \mid \psi^{-}\right\rangle} \in \mathcal{H}_{-}^{2},\left\langle\psi^{-} \mid \boldsymbol{q}[\mathrm{s}, 0]^{-}\right\rangle$ has the required analyticity properties on the complex s plane.

The function spaces (11) are dense in the Hilbert space and they can be equipped with a nuclear Frechét topology leading to a pair of rigged Hilbert spaces [15]. If we denote by $\Phi_{+}=\left\{\psi^{-}\right\}$and $\Phi_{-}=\left\{\phi^{+}\right\}$the set of vectors in $\mathcal{H}$ which have the $L^{2}$-realizations $(11 \pm$ ), then we have the pair of absract rigged Hilbert spaces

$$
\Phi_{ \pm} \subset \mathcal{H} \subset \Phi_{ \pm}^{\times}
$$

where $\Phi_{ \pm}^{\times}$are the spaces of continuous antilinear functionals on $\Phi_{ \pm}$. Then, the generalized eigenvectors $\left|\boldsymbol{q}[\mathrm{s}, 0]^{ \pm}\right\rangle$are defined as elements of $\Phi_{\mp}^{\times}$and the Dirac basis vector expansions hold for $\phi^{+}$and $\psi^{-}$(Gel'fand-Maurin theorem [7]).

With (11) and Laurent expansion of $S_{0}(\mathrm{~s})$ around $\mathrm{s}=\mathrm{s}_{R}$, we obtain the contour deformation of (10) over s

$$
\begin{aligned}
\left(\psi^{-}, \phi^{+}\right)= & \int \frac{\mathrm{d} \boldsymbol{q}}{2 q^{0}}\left\langle\psi^{-} \mid \boldsymbol{q}\left[\mathrm{s}_{R}, 0\right]^{-}\right\rangle\left\langle\boldsymbol{q}\left[\mathrm{s}_{R}, 0\right]^{+} \mid \phi^{+}\right\rangle \\
& +\int_{-\infty}^{\mathrm{s}_{0}} \int \frac{\mathrm{d} \boldsymbol{q}}{2 q^{0}}\left\langle\psi^{-} \mid \boldsymbol{q}[\mathbf{s}, 0]^{-}\right\rangle S_{0}(\mathrm{~s})\left\langle\boldsymbol{q}[\mathbf{s}, 0]^{+} \mid \phi^{+}\right\rangle .
\end{aligned}
$$


Like $\left|\boldsymbol{q}[\mathrm{s}, 0]^{-}\right\rangle$, the vectors $\left|\boldsymbol{q}\left[\mathbf{s}_{R}, 0\right]^{-}\right\rangle$appearing in (13) are well defined as elements of the dual space $\Phi_{+}^{\times}$. They are generalized eigenvectors of the momenta $P_{\mu}$ with complex eigenvalues $\sqrt{\mathrm{s}_{R}} q_{\mu}$ and for this reason, we call them Gamow vectors. The linear span of Gamow vectors for $\boldsymbol{q} \in \mathbb{R}^{3}$ defines the vector space that provides a state vector description for the resonance.

In $L^{2}\left(\mathbb{R}_{\mathrm{s}_{0}}, \mathbb{R}^{3}\right)$, the $U(\Lambda, a)$ act as $(U(\Lambda, a) f)(\mathrm{s}, \boldsymbol{q})=\mathrm{e}^{-\mathrm{i} \sqrt{\mathrm{s}} q \cdot a} f(\mathrm{~s}, \boldsymbol{\Lambda} \boldsymbol{q})$. Since $q$ remains real, it follows from this and the properties of Hardy class functions that the subspace (11-) of $L^{2}\left(\mathbb{R}_{\mathbf{s}_{0}}, \mathbb{R}^{3}\right)$ is invariant under $U(\Lambda, a)$ only if $a_{0} \geq 0$ and $a^{2} \geq 0$, i.e., only if $a$ is a translation into the forward light cone, $T_{+}$. The set of elements $\mathcal{P}_{+}:=\left\{(\Lambda, a) ;(\Lambda, a) \in \mathcal{P}, a \in T_{+}\right\}$is a semigroup under the product rule of $\mathcal{P}$. We call $\mathcal{P}_{+}$the causal Poincaré semigroup. Since $\Phi_{-}$is isomorphic to (11-), we conclude that the restriction of the unitary representation $U$ in $\mathcal{H}$ to $\Phi_{-}$ furnishes a representation of the semigroup $\mathcal{P}_{+}$. The restriction of the operators $U^{\dagger}$ to $\Phi_{+}$also provides a representation of $\mathcal{P}_{+}$different from that in $\Phi_{-}$.

By duality, the representations of $\mathcal{P}_{+}$in $\Phi_{ \pm}$induce representations in $\Phi_{ \pm}^{\times}$. In particular, the vectors $\left|\boldsymbol{q}[\mathrm{s}, 0]^{-}\right\rangle \in \Phi_{+}^{\times}$and $\left|\boldsymbol{q}\left[\mathrm{s}_{R}, 0\right]^{-}\right\rangle \in \Phi_{+}^{\times}$transform as

$$
\begin{aligned}
U(\Lambda, a)\left|\boldsymbol{q}[\mathrm{s}, 0]^{-}\right\rangle & =\mathrm{e}^{-\mathrm{i} \sqrt{\mathrm{s}} q \cdot a}\left|\boldsymbol{\Lambda} \boldsymbol{q}[\mathrm{s}, 0]^{-}\right\rangle, & & (\Lambda, a) \in \mathcal{P}_{+} \\
U(\Lambda, a)\left|\boldsymbol{q}\left[\mathrm{s}_{R}, 0\right]^{-}\right\rangle & =\mathrm{e}^{-\mathrm{i} \sqrt{\mathrm{s}_{R}} q \cdot a}\left|\boldsymbol{\Lambda} \boldsymbol{q}\left[\mathrm{s}_{R}, 0\right]^{-}\right\rangle, & & (\Lambda, a) \in \mathcal{P}_{+} .
\end{aligned}
$$

The representation of $\mathcal{P}_{+}$defined by (15) on the space spanned by $\left|\boldsymbol{q}\left[\mathrm{s}_{R}, 0\right]^{-}\right\rangle$ is irreducible. Therefore, in the same vein as stable elementary systems are represented by the UIR of $\mathcal{P}$, the irreducible representation of $\mathcal{P}_{+}$defined by (15) provides a characterization of the resonance associated with $\mathrm{s}_{R}$ as an elementary quantum system in its own right. It is noteworthy that although the restriction of $U$ to $\Phi_{-}$yields only a representation of $\mathcal{P}_{+}$, the restriction of the corresponding Lie algebra to $\Phi_{-}$yields a representation of the Poincaré algebra by continuous, interaction-incorporating operators. While the operator Lie algebra in $\mathcal{H}$ integrates to a representation of the whole group $\mathcal{P}$, only a cone of the Lie algebra in $\Phi_{-}$integrates to a representation of the semigroup $\mathcal{P}_{+}$in $\Phi_{-}$. The transformation properties of $\left|\boldsymbol{q}\left[\mathrm{s}_{R}, 0\right]^{-}\right\rangle$provide a unique, unambiguous criterion for extracting the resonance mass and width values from the pole position [3].

\section{Conclusion}

The BT-construction provides a way of introducing interactions into relativistic quantum systems without the use of fields that mediate them. We have shown that the BT construction can be extended to describe resonance states formed in 
two particle scattering. The key elements of the construction are the inclusion of interactions in the mass operator (7) and the use of Hardy class functions (11). From these considerations, we deduce that there exists an irreducible representation of the causal Poincaré semigroup uniquely characterized by the resonance pole position $\mathrm{s}_{R}$ and spin value $j$ of the partial S-matrix $S_{j}(\mathrm{~s})$. The use of Poincaré semigroup for describing resonances has been also considered in [14], though the interacting generators and the dynamical character of resonance formation has not been studied in this work. The same semigroup appears in [1] that uses the instant form dynamics and quantum fields to describe the interactions.

\section{Acknowledgments}

The author would like to acknowledge gratefully quite fruitful discussions with Professor W. N. Polyzou and financial support from Grinnell College.

\section{References}

[1] Antoniou I., Gadella M., Prigogine I. and Pronko G., Relativistic Gamow Vectors, J. Math. Phys. 39 (1998) 2995-3018.

[2] Bakamijian B. and Thomas L., Relativistic Particle Dynamics II , Phys. Rev. 92 (1953) 1300-1310.

[3] Bohm A. and Harshman N., On the Mass and Width of the Z-boson and Other Relativistic Quasistable Particles, Nucl. Phys. B 581 (2000) 91-115; Bohm A. and Sato Y., Relativistic Resonances: Their Masses, Widths, Lifetimes, Superposition, and Causal Evolution, Phys. Rev. D 71 (2005) 085018 (22 pages).

[4] Dirac P., Forms of Relativistic Dynamics, Rev. Mod. Phys. 21 (1949) 392399.

[5] Eden R., Landshoff P., Olive P. and Polkinghorne J., The Analytic S-Matrix, Cambridge University Press, Cambridge, 1966.

[6] Foldy L., Relativistic Particle Systems with Interaction, Phys. Rev. 122 (1961) 275-288; Coester F. and Polyzou W., Relativistic Quantum Mechanics of Particles with Ddirect Interactions, Phys. Rev. D 26 (1982) 13481367.

[7] Gel'fand I. and Vilenkin N., Generalized Functions, vol. 4, Academic Press, New York, 1964. 
[8] Kiester B. and Polyzou W., Relativistic Hamiltonian Dynamics in Nuclear and Particle Physics, in: Advances in Nuclear Physics, vol. 20, W. Negele and E. Vogt, (Eds.), Plenum, 1991.

[9] Koosis P., Introduction to $\mathcal{H}^{p}$ Spaces, London Math. Soc. Lecture Notes, vol. 40, Cambridge University Press, Cambridge, 1980.

[10] Macfarlane A., Relativistic Partial Wave Analysis, Rev. Mod. Phys. 34 (1962) 41-55; Bohm A. and Kaldass H., Relativistic Partial-wave Analysis Using the Velocity Basis of the Poincaré Group, Phys. Rev. A 60 (1999) 4606-4615.

[11] Nelson E., Analytic Vectors, Ann. Math. 70 (1959) 572-615; Flato M., Simon J., Snellman H. and Sternheimer D., Simple Facts About Analytic Vectors and Integrability, Ann. Scient. Ec. Norm. Sup. 4 (1972) 423-434; Wickramasekara S. and Bohm A., Symmetry Representations in the Rigged Hilbert Space Formulation of Quantum Mechanics, J. Phys. A: Math. Gen. 35 (2002) 807-829.

[12] Reed M. and Simon B., Scattering Theory, Academic Press, N.Y., 1979.

[13] Sokolov S., Hamiltonian Rrelativistic Quantum Description of System of Particles with Direct Interaction, Dokl. Akad. Nauk. USSR 233 (1977) 575578.

[14] Schulman L., Unstable Particles and the Poincaré Semigroup, Ann. Phys. 59 (1970) 201-218.

[15] Wickramasekara S., in preparation.

[16] Wigner E., On the Uunitary Representations of the Inhomogeneous Lorenz Group, Ann. Math. 40 (1939) 149-204.

Sujeev Wickramasekara

Department of Physics

Grinnell College

Grinnell, IA 50112

USA

E-mail address: wickrama@grinnell.edu 